\title{
RESIDUALLY FINITE ONE-RELATOR GROUPS
}

\author{
BY GILBERT BAUMSLAG ${ }^{1}$
}

Communicated by Michio Suzuki, May 16, 1967

Introduction. It seems to be commonly believed that the presence of elements of finite order in a group with a single defining relation is a complicating rather than a simplifying factor. This note is in support of the opposite point of view, lending respectability to the

CONJECTURE A. Every group with a single defining relation with nontrivial elements of finite order is residually finite.

In order to put our results in their proper setting let us define $\langle l, m\rangle$ to be the group generated by $a$ and $b$ subject to the single defining relation $a^{-1} b^{l} a b^{m}=1$ :

$$
\langle l, m\rangle=\left(a, b ; a^{-1} b^{l} a b^{m}=1\right) .
$$

Adding a third parameter we define

$$
\langle l, m ; t\rangle=\left(a, b ;\left(a^{-1} b^{l} a b^{m}\right)^{t}=1\right) .
$$

Let $\&$ be the class of those groups $\langle l, m\rangle$ satisfying $|l| \neq 1 \neq|m|, l m \neq 0$, and $l$ and $m$ relatively prime. Furthermore, let $\mathfrak{T}$ be the class of these groups $\langle l, m ; t\rangle$ satisfying the conditions imposed above on $l$ and $m$, and in addition the extra two conditions $t>1$, and $l, m$ and $t$ relatively prime in pairs. The point of our initial remark is that $\mathfrak{T}$ looks more complicated than $\mathscr{L}$. Actually $\mathscr{\&}$ is quite a nasty class of groups. Indeed the main result of [1] is that every group in $\&$ is isomorphic to one of its proper factor groups, i.e. nonhopfian. Since finitely generated residually finite groups are hopfian (A. I. Mal'cev [2]) no group in $\&$ is residually finite. Our contribution to Conjecture A is that the groups in $\mathfrak{T l}$ are residually finite.

THEOREM 1. Every group in the class $\mathrm{T}$ is residually finite.

In fact even more is true.

THEOREM 2. If $l, m$, t are relatively prime in pairs $(l \neq 0 \neq m)$ and if $t$ is a power of a prime $p(t \neq 1)$ then the group $\langle l, m ; t\rangle$ is residually $a$ finite p-group.

Conjecture A seems difficult. A somewhat easier related conjecture is

${ }^{1}$ Support from the National Science Foundation is gratefully acknowledged. The author is a Sloan Fellow. 
CONJECTURE Ab. Every finitely generated group with a single defining relation with nontrivial elements of finite order is hopfian.

The theory of groups with a single defining relation has been developed sufficiently for us to be able to prove

THEOREM 3. Let $G$ be a group with a single defining relation and let $T$ be the subgroup of $G$ generated by the elements of finite order. If $G / T$ is hopfian, so is $G$.

The existence of the nonhopfian group $\langle 2,3\rangle$ together with Theorem 1 show that the converse of Theorem 3 is false. This underlines to some extent the difficulties involved in the proof of Theorem 1.

Remarks on the proofs. The proof of Theorem 1 goes as follows. Suppose $G \in \mathscr{T}$. Thus

$$
G=\left(a, b ;\left(a^{-1} b^{l} a b^{m}\right)^{t}=1\right) .
$$

We observe that if $N$ is the normal closure of $b$ in $G$ then $G / N$ is infinite cyclic. Our procedure is to prove that $N$ is residually finite. Since an extension of a residually finite group by another residually finite group need not be residually finite we have to establish that $N$ is residually finite in such a way that we are able to deduce the residual finiteness of $G$. To establish the results we need about $N$ we have to obtain sufficient information about certain one-relator subgroups from which $N$ is constructed. This information is contained in the following lemmas.

LEMMA 1. The groups

$$
\left(a, b ;\left(a^{l} b^{m}\right)^{t}=1\right) \quad(t>1)
$$

contain a normal subgroup of finite index which is residually free.

Lemma 2. The groups

$$
\left(a, b ;\left(a^{l} b^{m}\right)^{t}=1\right) \quad(t>1)
$$

are residually finite p-groups if $t$ is a power of the prime $p$.

Both Lemma 1 and Lemma 2 make use of the ReidemeisterSchreier procedure for finding generators and defining relations for a subgroup of a group given by generators and defining relations (see $[3$, p. 86]) as well as the main results of $[4]$ and $[5]$ on the residual properties of certain generalized free products.

The proof of Theorem 2 involves a refinement of the proof of Theorem 1 and an old theorem of $\mathrm{P}$. Hall, namely that an automorphism of 
a finite $p$-group $P$ which induces an automorphism of $p$-power order on $P$ modulo its frattini subgroup is itself of $p$-power order (see e.g. $[6$, p. 178]).

Finally the proof of Theorem 3 depends on the known structure of $T$ [7] and the fact that in a one-relator group every pair of elements of maximal finite order are conjugate [8].

Extension of results. Theorem 1 can be extended to certain groups with a single defining relation on more than two generators. At the present time I am unable to relax the conditions on $l, m$ and $t$ to $t>1$. But it is certainly likely that $\langle l, m ; t\rangle(t>1)$ is residually finite. This can probably be proved by similar arguments to those used in the proof of Theorem 1. A proof of Conjecture A, however, at this time, seems out of reach.

\section{REFERENCES}

1. G. Baumslag and D. Solitar, Some two-generator one-relator non-hopfian groups, Bull. Amer. Math. Soc. 68 (1962), 199-201.

2. A. I. Mal'cev, On isomorphic representations of infinite groups by matrices, Mat. Sb. 8 (1940), 405-422.

3. W. Magnus, A. Karrass and D. Solitar, Combinatorial group theory, Interscience, New York, 1966.

4. G. Baumslag, On generalized free products, Math. Z. 78 (1962), 423-438.

5. - On the residual nilpotence of certain one-relator groups, J. Algebra (to appear).

6. M. Hall, The theory of groups, Macmillan, New York, 1959.

7. D. E. Cohen and R. C. Lyndon, Free bases for normal subgroups of free groups, Trans. Amer. Math. Soc. 108 (1963), 526-537.

8. A. Karrass, W. Magnus and D. Solitar, Elements of finite order in groups with a single defining relation, Comm. Pure Appl. Math. 13 (1960), 57-66.

Graduate Center, The City University of New York 